\title{
Best Fredholm perturbation theorems
}

by

\section{SCHECHTER and ROBERT WHITLEY (Irvine, Cal.)}

Abstract. Consider a basic Fredholm perturbation theorem; for example:

Let $T$ be a Fredholm operator and suppose that $B$ is a linear operator with $\|B\|<\gamma(T)$.

Then $T+B$ is a Fredholm operator with the same index as $T$.

The question we consider, for this theorem and others like it, is to what extent can the functions $\|B\|, \gamma(T)$, and $\|B\| / \gamma(T)$ be improved and still have the conclusions of the theorem hold.

I. Basic theorems. The problem of finding the "best possible" constants in perturbation theorems has been studied by many authors. In this first section it is shown that for two classical perturbation theorems it is possible to describe the "best" perturbation constant for that theorem. These specific results raise the question dealt with in the rest of the paper: What is the general form of a perturbation theorem and what constitutes a best theorem.

One concrete instance of the problem of finding a best perturbation constant is that of finding the best constant $\gamma(T)$ for the classical Theorem A below; this question is raised in [8, p. 96] for a weaker version of Theorem $A$. The answer to this question is given as Theorem 1 below, and was given in [11] for operators with a bounded inverse.

Here, and in what follows, all operators are bounded linear operators mapping a Banach space $X$ into a Banach space $Y, \alpha(T)$ is the dimension of the null space $N(T)$ of $T$, and $\beta(T)$ is the codimension of the range of T. A Fredholm operator $T$ has both $\alpha(T)$ and $\beta(T)$ finite, and its index is $x(T)=\alpha(T)-\beta(T)$.

The perturbation constant $\gamma(T)$ used in [8] depends upon the choice of complementary summands. We will instead here let $\gamma(T)$ denote Kato's minimum modulus $[10$, p. 231 or 6, p. 96] defined by

$$
\gamma(T)=\inf \{\|T x\| / d(x, N(T)): d(x, N(T))>0\} .
$$

The operator $T$ has closed range iff $\gamma(T)>0[6$, p. 98].

The $\Phi_{+}$operators of Gohberg and Krein [7] are defined by: an operator $T$ is a $\Phi_{+}$operator if it has closed range and finite nullity $\alpha(T)$.

THEOREM A. For any $\Phi_{+}$operator $T$, if $B$ is a linear operator with $\|B\|<\gamma(T)$, then $T+B$ is a $\Phi_{+}$operator with:

(i) $x(T+B)=x(T)$.

(ii) $\alpha(T+B) \leqslant \alpha(T)$. 
The index $\varkappa(T)$ of a $\Phi_{+}$operator $T$ may be $-\infty$. A Fredholm operator is a $\Phi_{+}$operator, since $T$ has closed range if $\beta(T)$ is finite [6, p. 100].

Kato's perturbation constant $\gamma(T)$ is best possible for Theorem A in the following sense.

1. THEOREM. Let $T$ be a $\Phi_{+}$operator on an infinite-dimensional Banach space. Given $\varepsilon>0$ there is an operator $B$ with $\|B\|<\gamma(T)+\varepsilon$, but $\alpha(T+B)>\alpha(T)$.

Proof. Let $N=N(T)$. Given $\varepsilon>0$ there is a vector $v$ with $d(v, N)=1$ and $\|T v\|<\gamma(T)+\varepsilon$. Since $N$ is finite-dimensional, there is a $w$ with $w+N=v+N$ and $\|w\|=d(w, N)=1$. Let $x^{*}$ be a linear functional with $x^{*}(w)=1, x^{*}(N)=0$, and $\left\|x^{*}\right\|=1$. Define

$$
B x=-x^{*}(x) T w
$$

and note that $\|B\|=\left\|x^{*}\right\|\|T w\|<\gamma(T)+\varepsilon$. The null space of $T+B$ is $N^{\prime}=N+\operatorname{sp}(w)$. To see this, it is clear that $N(T+B)$ contains $N^{\prime}$, and if $(T+B) x=0$, then $T\left(x-x^{*}(x) w\right)=0$ and $x$ belongs to $N^{\prime}$. Hence $\alpha(T+B)=\alpha(T)+1$.

The other classical perturbation theorem we will consider originated in classical spectral theory for a compact operator $K$, in which one concludes that $\lambda I-K$, for $\lambda \neq 0$, is a Fredholm operator with index zero, but with nullity which, though finite, may be nonzero. More generally, one concludes, under appropriate hypotheses, that $T+B$ is $\Phi_{+}$with $\varkappa(T+B)=\varkappa(T)$, but conclusion (ii) of Theorem A is omitted. For this theorem we define an analogue to $\gamma(T)$.

\section{DeFINITION.}

$$
\mu(T)=\inf \{\|A\|: \alpha(T-A)=\infty\} .
$$

Here we presuppose that Banach spaces $X$ and $Y$ are given and that $T$ as well as all the operators $A$ over which the infimum in (3) is taken, map $X$ into $Y$. From now on, $X$ will always be infinite-dimensional, and all subspaces will be closed infinite-dimensional subspaces.

The next lemma will be used repeatedly.

3. Lemma. An operator $A$ is not a $\Phi_{+}$operator iff for each $\varepsilon>0$ there is a compact operator $K$ with $\|K\|<\varepsilon$ and $\alpha(A-K)=\infty$. Consequently, $T$ is a $\Phi$ operator iff $\mu(T)>0$.

Proof. The first part of the lemma follows from [15, Theorem 23] or $[6, \mathrm{p} .80]$. This result and (3) together imply that $\mu(T)=0$ if $T$ is not $\Phi_{+}$. If $T$ is $\Phi_{+}$then there is a (closed) subspace $M^{\prime}$ of finite codimension with the restriction of $T$ to $M^{\prime},\left.T\right|_{M^{\prime}}$, one-to-one with closed range. Thus for all $x$ in $M^{\prime}$, $\|T x\| \geqslant \gamma\|x\|$, with $\gamma=\gamma\left(\left.T\right|_{M^{\prime}}\right)$. Then if for some $A, \alpha(A-T)=\infty$, there is an (infinite-dimensional closed) subspace $M$ with $T=A$ on $M$, and then $\|A\| \geqslant\left\|\left.T\right|_{M}\right\| \geqslant\left\|\left.T\right|_{M \cap M^{\prime}}\right\| \geqslant \gamma$. Therefore $\mu(T) \geqslant \gamma$.
4. THEOREM. Let $T$ be a $\Phi_{+}$operator. If $B$ is an operator with (4)

$$
\|B\|<\mu(T)
$$

then $T+B$ is a $\Phi_{+}$operator with $\chi(T+B)=\chi(T)$. Further, given $\varepsilon>0$ there is a $B$ with $\|B\|<\mu(T)+\varepsilon$, but $T+B$ is not a $\Phi_{+}$operator.

Pro of. If $T+B$ is not a $\Phi_{+}$operator, then, given $\varepsilon>0$, by Lemma 3 there is a $K$ with $\|K\|<\varepsilon$ and $\alpha(T+B-K)=\infty$. Then $\mu(T) \leqslant\|B-K\| \leqslant\|B\|+\varepsilon$, and (4) cannot hold. Lemma 6 of the next section shows that $x(T+B)=x(T)$.

By the definition of $\mu(T)$, given $\varepsilon>0$ there is a $B$ with $\|B\|<\mu(T)+\varepsilon$ and $\alpha(T-B)=\infty$.

If $T$ is a $\Phi_{+}$operator, then Theorem 4 shows that the function $\mu(T)$ is the same as the distance from $T$ to the complement of the $\Phi_{+}$operators which was studied in [22] and [23].

Theorems 1 and 2 show that the functions $\gamma(T)$ and $\mu(T)$ are, in one sense, best possible for their respective theorems, in which we use $\|B\|$ to measure the size of $B$. The question of how $\|B\|$ could be improved is one of a class of questions which will be discussed in the following sections.

\section{Perturbation functions}

5. Defintition. Let $m(B, T)$ be a nonnegative function, defined for any operator $B$ and any $T$ either a $\Phi_{+}$operator, in (a) and (c) below, or a $\Phi$ (= Fredholm) operator, in (b) below.

(a) The function $m(B, T)$ is a $\Phi_{+}$perturbation function if:

(i) $m(\lambda B, T)=|\lambda| m(B, T)$

(ii) $m(B, T)<1$ for $T \Phi_{+}$implies $T+B$ is $\Phi_{+}$.

(b) Similarly, $m(B, T)$ is a $\Phi$ (or Fredholm) perturbation function if:

(i) $m(\lambda B, T)=|\lambda| m(B, T)$.

(ii) $m(B, T)<1$ for $T \Phi$ implies $T+B$ is $\Phi$.

(c) Also, $m(B, T)$ is a $\Phi_{\alpha}$ perturbation function if:

(i) $m(\lambda B, T)=|\lambda| m(B, T)$.

(ii) $m(B, T)<1$ for $T \Phi_{+}$implies $T+B$ is $\Phi_{+}$and $\alpha(T+B) \leqslant \alpha(T)$.

The phrase "a perturbation function" will ambiguously refer to one of these three types described above.

Perturbation functions are defined for operators mapping a (infinite-dimensional) Banach space $X$ into a Banach space $Y$. This dependence on $X$ and $Y$ is not made explicit in the notation $m(B, T)$, but whenever a perturbation function is discussed, all the operators which occur in the discussion will be maps from $X$ into $Y$.

Theorem A shows that $\|B\| / \gamma(T)$ is a $\Phi_{\alpha}$ perturbation function, and therefore also a $\Phi_{+}$perturbation function. Theorem 4 shows that $\|B\| / \mu(T)$ is a $\Phi_{+}$perturbation function. One consequence of Lemma 6 , below, is that a $\Phi_{+}$ perturbation function is also a $\Phi$ perturbation function when restricted to Fredholm operators $T$. 
6. LEMMA. Let $m(B, T)$ be a perturbation function. If $m(B, T)<1$, then $x(T+B)=x(T)$.

Proof. In the case that $m(B, T)$ is either a $\Phi_{+}$p.f. or a $\Phi_{\alpha}$ p.f., take $T$ to be a $\Phi_{+}$operator; in the case that $m(B, T)$ is a $\Phi$ p.f., take $T$ to be a $\Phi$ operator.

The following technique is standard $[6, \mathrm{p} .118]$. Consider the function $f(\lambda)=x(T+\lambda B)$, defined for $\lambda$ in $[0,1]$. To see that $f$ is continuous, let $\lambda^{\prime}$ in $[0,1]$ be given. Since $m\left(\lambda^{\prime} B, T\right) \leqslant m(B, T)<1, T+\lambda^{\prime} B$ is a $\Phi_{+}$operator with $\gamma=\gamma\left(T+\lambda^{\prime} B\right)>0$. If $\left|\lambda-\lambda^{\prime}\right|\|B\|<\gamma^{\prime}$, then $\varkappa(T+\lambda B)=\varkappa\left(T+\lambda^{\prime} B\right)$ by Theorem $A$. Since the range of $f$ is the integers, together with $-\infty$, with the discrete topology, $f$, being continuous, must be constant with $f(0)=x(T)=f(1)$ $=x(T+B)$.

The injection modulus $j(T)$ of an operator $T[13$, p. 26] is defined by

$$
j(T)=\sup \{\lambda:\|T x\| \geqslant \lambda\|x\| \text { for all } x\} .
$$

If $T$ is one-to-one $j(T)=\gamma(T)$, whereas $j(T)=0$ if $T$ is not one-to-one.

The four functions below were introduced in [15]. In the definitions, and elsewhere, $M$ and $N$ denote closed infinite-dimensional subspaces of the domain space $X$.

$$
\begin{aligned}
\Gamma(T) & =\inf \left\{\left\|\left.T\right|_{M}\right\|: M\right\}, \\
\Delta(B) & =\sup \left\{\Gamma\left(\left.B\right|_{M}\right): M\right\}, \\
\nu(T) & =\sup \left\{j\left(\left.T\right|_{M}\right): \operatorname{cod} M<\infty\right\}, \\
\tau(B) & =\sup \left\{j\left(\left.B\right|_{M}\right): M\right\} .
\end{aligned}
$$

In [14] it is shown that $T$ is a $\Phi_{+}$operator iff $\Gamma(T)>0$ iff $v(T)>0$, and that if $T$ is a $\Phi_{+}$operator and either $\Delta(B)<\Gamma(T)$ or $\tau(B)<v(T)$, then $T+B$ is a $\Phi_{+}$operator. To use the terminology of Definition $5, \Delta(B) / \Gamma(T)$ and $\tau(B) / v(T)$ are $\Phi_{+}$perturbation functions. Also see Theorems 5.1 and 5.2 of [22].

If $m_{1}(B, T)$ is a perturbation function and $m_{2}(B, T)$ is a function satisfying $m_{2}(\lambda B, T)=|\lambda| m_{2}(B, T)$, then the inequality $m_{1}(B, T) \leqslant m_{2}(B, T)$, for all $B$ and all suitable $T$, implies that $m_{2}(B, T)$ is itself a perturbation function. Thus one corollary of Lemma 9 below is that $\Delta(B) / \Gamma(T)$ and $\tau(B) / v(T)$ are $\Phi_{+}$ perturbation functions.

8. Definition. For $B$ an operator and $T$ a $\Phi_{+}$operator define (10)

$\varrho(B, T)=\inf \{\sup \{\inf (\|B x\| /\|T x\|: T x \neq 0, x$ in $N): N \subset M\}: \operatorname{cod} M<\infty\}$.

9. Lemma. The function $\varrho(B, T)$ is a $\Phi_{+}$perturbation function with

$$
\varrho(B, T) \leqslant \Delta(B) / \Gamma(T),
$$

Proof. Suppose that $\varrho(B, T)<a<1$. There is an $M$ of finite codimension, on which $T$ is one-to-one, such that for each $N \subset M$ there is a norm one $x^{\prime}$ in $N$ with $\left\|B x^{\prime}\right\| /\left\|T x^{\prime}\right\|<a$. Assume that $T+B$ is not $\Phi_{+}$, and therefore that $T+B$ is not $\Phi_{+}$when restricted to $M$. By Lemma 3 there is an $N \subset M$ with

$$
\|T x\| \leqslant\|B x\|+\varepsilon\|x\| j\left(\left.T\right|_{M}\right)
$$

for all $x$ in $N$ and any preassigned positive $\varepsilon$. For $x^{\prime}$ chosen in $N$ as above,

$$
\left\|T x^{\prime}\right\|<a\left\|T x^{\prime}\right\|+\varepsilon j\left(\left.T\right|_{M}\right),
$$

that is,

$$
\varepsilon j\left(\left.T\right|_{M}\right)>(1-a)\left\|T x^{\prime}\right\| \geqslant(1-a) j\left(\left.T\right|_{M}\right)
$$

which is impossible for small $\varepsilon$. Thus $T+B$ is $\Phi_{+}$and therefore $\varrho(B, T)$ is a $\Phi_{+}$ perturbation function.

Given $N$, set $\inf \{\|T x\| /\|B x\|: x$ in $N\}=1 / c$, which may be $+\infty$, so that for $x$ in $N$,

$$
c\|T x\| \leqslant\|B x\| .
$$

Then $c\left\|\left.T\right|_{L}\right\| \leqslant\left\|\left.B\right|_{L}\right\|$ for $L \subset N$, and so $c \Gamma\left(\left.T\right|_{L}\right) \leqslant \Gamma\left(\left.B\right|_{L}\right)$. Thus

$$
\varrho(B, T) \leqslant \inf \left\{\sup \left\{\Gamma\left(\left.B\right|_{N}\right) / \Gamma\left(\left.T\right|_{N}\right): N \subset M\right\}: \operatorname{cod} M<\infty\right\}
$$

$$
\leqslant \inf \left\{\Delta\left(\left.B\right|_{M}\right) / \Gamma\left(\left.T\right|_{M}\right)\right\} \leqslant \Delta(B) / \Gamma(T) .
$$

(13) implies that $c j\left(\left.T\right|_{N}\right) \leqslant j\left(\left.B\right|_{N}\right)$ and therefore

$$
\begin{aligned}
\varrho(B, T) & \leqslant \inf \left\{\sup \left\{j\left(\left.B\right|_{N}\right) / j\left(\left.T\right|_{N}\right): N \subset M\right\}: \operatorname{cod} M<\infty\right\} \\
& \leqslant \inf \left\{\tau\left(\left.B\right|_{M}\right) / j\left(\left.T\right|_{M}\right): \operatorname{cod} M<\infty\right\} \leqslant \tau(B) / v(T) .
\end{aligned}
$$

Since $\varrho(B, T)$ is smaller than the perturbation functions in (11) and (12), it is better in the most obvious sense. For perturbation functions, smaller is better and smallest is best, for which see Lemma 10 below.

10. LeMMA. There is a smallest perturbation function. In fact:

(i) The smallest $\Phi_{+}$perturbation function is

$$
m_{1}(B, T)=\max \left\{|\lambda|: \lambda T+B \text { is not } \Phi_{+}\right\} .
$$

(ii) The smallest $\Phi$ perturbation function is

$$
m_{2}(B, T)=\max \{|\lambda|: \lambda T+B \text { is not } \Phi\} .
$$

(iii) The smallest $\Phi_{\alpha}$ perturbation function is

(16) $\quad m_{3}(B, T)=\max \left\{|\lambda|\right.$ : either $\lambda T+B$ is not $\Phi_{+}$or $\left.\alpha(\lambda T+B)>\alpha(T)\right\}$. Proof. It is immediate from (14), (15) and (16) that each of $m_{1}, m_{2}$, and $m_{3}$ is a p.f. 
If $m(B, T)$ is a $\Phi_{+}$p.f. and $m(B, T)<m_{1}(B, T)$ for some $B$ and some $\Phi_{+} T$, then by (14) there is a $\lambda^{\prime}$ satisfying $m(B, T)<\left|\lambda^{\prime}\right|$ with $\lambda^{\prime} T+B$ not a $\Phi_{+}$ operator. But this is a contradiction because $m\left(B / \lambda^{\prime}, T\right)<1$, so $T+B / \lambda^{\prime}$, and therefore $\lambda^{\prime} T+B$ is a $\Phi_{+}$operator.

Similar arguments show that $m_{2}$ and $m_{3}$ are minimal.

The point of Lemma 10 is that there are minimal perturbation functions. The formulas (14), (15), and (16) beg the question of whether, for given $T$ and $B$, $T+B$ has the desired property. Therefore while it is not possible to have, say, a better $\Phi$ perturbation function than $m_{2}$, it is certainly possible to have a formula for $m_{2}$ which is more informative than (15).

Recall that an operator $T$ is Fredholm iff there is an operator $T_{1}$ with $T T_{1}=I+K_{1}$ on $X$ and $T_{1} T=I+K_{2}$ on $Y$, where $K_{1}$ and $K_{2}$ are compact $[16$, p. 108].

Let $\pi$ denote the quotient map of the bounded linear operators on $X, \mathscr{B}(X)$, onto $\mathscr{B}(X) / \mathscr{C}(X), \mathscr{C}(X)$ the compact operators on $X$. For an operator $T$ on $X$ let $r_{\sigma}(\pi(T))$ be the spectral radius of the element $\pi(T)$ in the Banach algebra $\mathscr{B}(X) / \mathscr{C}(X)$. Compare with Theorem 5 of [23].

11. THEOREM. Let $T$ be Fredholm, and suppose that $A$ is an operator, like $T_{1}$ above, for which $A T=I+K_{1}$ and $T A=I+K_{2}, K_{1}$ and $K_{2}$ compact. Then, setting $C=A B$, the best Fredholm perturbation function $m_{2}$ is given by

$$
\begin{aligned}
& m_{2}(B, T)=\lim \left(\tau\left(C^{n}\right)\right)^{1 / n}, \\
& m_{2}(B, T)=\lim \left(\Delta\left(C^{n}\right)\right)^{1 / n}, \\
& m_{2}(B, T)=r_{\sigma}(\pi(C)) .
\end{aligned}
$$

Proof. Since $A$ is also Fredholm, $\lambda T+B$ is Fredholm iff $A(\lambda T+B)$ $=\lambda I+A B+\lambda K_{1}$ is Fredholm iff $\lambda I+A B$ is Fredholm. Thus (19) follows from the equation $r_{\sigma}(\pi(C))=\sup \{|\lambda|: \lambda I-C$ is not in $\Phi\}$.

In $\left[14\right.$, p. 1062] it was shown that $r_{\sigma}(\pi(C))=\lim \left(\tau\left(C^{n}\right)\right)^{1 / n}=\lim \left(\Delta\left(C^{n}\right)\right)^{1 / n}$ the lim being taken, of course, as $n$ tends to infinity.

A technical detail bars the extension of Theorem 11 to $\Phi_{+}$perturbations and $m_{1}$. Namely, an operator $T$ in $\Phi_{+}$may not have a complemented range. One must consider, instead of $\Phi_{+}$operators, the class $\Phi^{l}$ of those operators in $\Phi_{+}$which do have complemented ranges [21], and use the fact that $T$ belongs to $\Phi^{l}$ iff there is an operator $T_{1}$ with $T_{1} T=I+K, K$ compact. We do not state this extension.

Note that if $m(B, T)$ is a Fredholm perturbation function and $T$ a Fredholm operator with $A$ an operator as in Theorem 11, then $m^{\prime}(B, T)=m(A B, I)$ is a Fredholm perturbation function. function.

12. EXAmple. The function $\varrho(B, T)$ is not the smallest $\Phi_{+}$perturbation
Proof. Let $X=Y=l^{2}$, and define $B$ for $a=\sum \alpha_{j} e_{j}$ by $B a$ $=\alpha_{2} e_{3}+\alpha_{4} e_{5}+\ldots$ Let $N$ be the closed subspace spanned by $\left\{e_{2}, e_{4}, \ldots\right\}$. For $M$ a subspace of finite codimension, $M^{\prime}=N \cap M$ is infinite-dimensional, and $j\left(\left.B\right|_{M^{\prime}}\right)=1=\left\|\left.B\right|_{M^{\prime}}\right\|$, and so $\varrho(B, I)=1$. However, $(\lambda I+B)(\lambda I-B)=\lambda^{2} I$ since $B^{2}=0$, and thus $m_{3}(B, I)=m_{2}(B, I)=m_{1}(B, I)=0$.

Note that since $\Delta(B)=\tau(B)=1$, the perturbation functions $\Delta(B) / \gamma(I)$ and $\tau(B) / v(I)$ are both 1 .

13. LEMMA. Let $H$ be a Hilbert space, and suppose that $T: H \rightarrow H$ has $v\left(\left.T\right|_{M}\right)>0$. Given $\varepsilon>0$ there is an $N \subset M$ with $\left\|\left.T\right|_{N}\right\|<v\left(\left.T\right|_{M}\right)+\varepsilon$.

Proof. To simplify the notation, take $M=H$. From (8), given $\varepsilon>0$ there is an $M^{\prime}$ of finite codimension with $\nu\left(\left.T\right|_{M^{\prime}}\right)>0$, and therefore $T$ is $\Phi_{+}$and there is a norm one $x_{1}$ in $M^{\prime}$ with $\left\|T x_{1}\right\|<j\left(\left.T\right|_{M^{\prime}}\right)+\varepsilon \leqslant v(T)+\varepsilon$. Let $N$ be the orthogonal complement of $\operatorname{sp}\left(x_{1}\right), N^{\prime}$ be the orthogonal complement of $\operatorname{sp}\left(T x_{1}\right)$, and set $M^{\prime \prime}=N \cap T^{-1}\left(N^{\prime}\right) \cap M^{\prime}$, which has finite codimension in $M^{\prime}$. There is a norm one $x_{2}$ in $M^{\prime \prime}$ with $\left\|T x_{2}\right\|<j\left(\left.T\right|_{M^{\prime \prime}}\right)+\varepsilon \leqslant v(T)+\varepsilon$. Since $x_{2}$ is in $M^{\prime \prime}, x_{2}$ is orthogonal to $x_{1}$ and $T x_{2}$ is orthogonal to $T x_{1}$.

Continuing in this way we obtain an orthonormal sequence $\left\{x_{n}\right\}$ and an orthogonal sequence $\left\{T x_{n}\right\}$ with $\left\|T x_{n}\right\|<v(T)+\varepsilon$. On the closed span of the $\left\{x_{n}\right\}$,

$$
\left\|T \sum \alpha_{n} x_{n}\right\|^{2}=\sum\left|\alpha_{n}\right|^{2}\left\|T x_{n}\right\|^{2}<(v(T)+\varepsilon)\left\|\sum \alpha_{n} x_{n}\right\|^{2} .
$$

14. Theorem. On a Hilbert space, $\tau(B)=\Delta(B)$ and $v(T)=\Gamma(T)$; consequently, the two perturbation functions $\Delta(B) / \Gamma(T)$ and $\tau(B) / v(T)$ are equal.

Proof. To show that $\Delta(B) \leqslant \tau(B)$, choose an $M$ with $\Gamma\left(\left.B\right|_{M}\right)>0$. By [14], $v\left(\left.B\right|_{M}\right)>0$, so given $\varepsilon>0$ by Lemma 13 there is an $N$ contained in $M$ with $\left\|\left.B\right|_{N}\right\|<v\left(\left.B\right|_{M}\right)+\varepsilon \leqslant \tau\left(\left.B\right|_{M}\right)+\varepsilon \leqslant \tau(B)+\varepsilon$. Hence $\Gamma\left(\left.B\right|_{M}\right) \leqslant \tau(B)+\varepsilon$ and consequently $\Delta(B) \leqslant \tau(B)$. The reverse inequality is given in [14].

We know from [14] that $v(T) \leqslant \Gamma(T)$. The reverse inequality is well known [22, p. 229] and follows from Lemma 13

Note that $\Delta(T)$ is the Calkin norm of $T$, which follows from the submultiplicativity of $\Delta$ and [20].

15. EXAMPLE. Suppose that a Banach space $X$ has the following two properties.

(i) There is a strictly singular operator $S: X \rightarrow X$ which is not compact.

(ii) The space $X$ is isomorphic to its Cartesian square $X \times X$.

Examples of such spaces are $L^{p}[0,1]$ for $p \neq 2$, and $C[0,1]$.

Then there is an isomorphism T mapping $X$ onto $X$ with $v(T)<\Gamma(T)$.

Proof. We first construct such an operator on $Z=X \times X$, normed by, say, $\|(x, y)\|=\|x\|+\|y\|$. Define $V$ on $Z$ by $V(x, y)=(0, S x), S$ being the operator in condition (i). The map $V$ is strictly singular, being the composition 
of $S$ with two bounded operators, and is easily seen not to be compact [19]. Set $T=\lambda I-V$. Since $V^{2}=0,(\lambda I-V)(\lambda I+V)=\lambda^{2} I$, and $T$ is one-to-one and onto.

Since $V$ is strictly singular, given $M$ and $\varepsilon>0$ there is an $M^{\prime} \subset M$ with $\left\|\left.V\right|_{M^{\prime}}\right\|<\varepsilon[6$, p. 84]. From this it follows that $\Gamma(T)=|\lambda|$.

An operator $K$ is compact iff for each $\varepsilon>0$ there is an $M$ of finite codimension with $\left\|\left.K\right|_{M}\right\|<\varepsilon[6$, p. 85]. Because $S$ is not compact,

$$
a=\inf \left\{\left\|\left.S\right|_{M}\right\|: \operatorname{cod} M<\infty\right\}>0 .
$$

Let $M$ be a subspace of finite codimension in $Z$. There are linear functionals $z_{1}^{*}, \ldots, z_{m}^{*}$ with $M=\bigcap N\left(z_{n}^{*}\right)$. Each of these functionals can be written $z_{n}^{*}(x, y)=x_{n}^{*}(x)+y_{n}^{*}(y)$, where $x_{n}^{*}$ and $y_{n}^{*}$ are in the conjugate space $X^{*}$ of $X$ Set $M^{\prime}=\bigcap N\left(x_{n}^{*}\right)$, and note that $M^{\prime \prime}=M^{\prime} \times\{0\} \subset M$ so that $j\left(\left.T\right|_{M}\right) \leqslant$ $j\left(\left.T\right|_{M^{\prime \prime}}\right)$. Because $T$ has the inverse $\lambda^{-2}(\lambda I+V)$, it follows that $j\left(\left.T\right|_{M^{\prime \prime}}\right)=|\lambda|^{2}\left(\left\|\left.(\lambda I+V)\right|_{M^{\prime \prime}}\right\|\right)^{-1}$. Now

$\left\|\left.(\lambda I+V)\right|_{M^{\prime \prime}}\right\|=\sup \left\{\|\lambda x\|+\|S x\|:\|x\|=1\right.$ and $x$ in $\left.M^{\prime}\right\} \geqslant|\lambda|+a$.

Hence $j\left(\left.T\right|_{M}\right) \leqslant|\lambda|^{2} /(|\lambda|+a)$, and therefore

$$
\nu(T) \leqslant(|\lambda| /(|\lambda|+a)) \Gamma(T)
$$

This gives the desired example on $Z$.

Let $U: X \rightarrow Z$ be an isomorphism of $X$ with $Z$, as hypothesized in condition (ii), and set $T^{\prime}=U^{-1} T U$. It is easy to show that $v\left(T^{\prime}\right) \leqslant b v(T)$ and $\Gamma(T) \leqslant b \Gamma\left(T^{\prime}\right)$, where $b=\left\|U^{-1}\right\|\|U\|$. Hence

$$
v\left(T^{\prime}\right) \leqslant b^{2}(|\lambda| /(|\lambda|+a)) \Gamma\left(T^{\prime}\right)
$$

and the R.H.S. is less than $\Gamma\left(T^{\prime}\right)$ for $\lambda$ small.

There are strictly singular operators which are not compact on $L^{p}[0,1]$ for $p \neq 2$ (see [7]) and on $C[0,1]$ (see [12]). The fact that all the common Banach spaces are isomorphic to their Cartesian square goes back to Banach ([1, pp. 244-5]; see also [2]).

III. Factored perturbation functions. Given a $\Phi_{+}$operator $T$ and an operator $B$, the computation of $\|B\| / \gamma(T)$ involves the computation of the two useful numbers $\|B\|$ and $\gamma(T)$. After this computation, $\gamma(T)$ can, of course, be used in studying the perturbation of $T$ by other operators and $\|B\|$ can be used in studying the perturbation of other $\Phi_{+}$operators by $B$. In contrast, the computation of, say, $\varrho(B, T)$ must be completely redone if either $B$ or $T$ changes. This observation leads to Definition 16. the form

6. Definition. A perturbation function $m$ is factored if it can be written in

$$
m(B, T)=m_{1}(B) / m_{2}(T)
$$

17. THEOREM. Let the factored perturbation function.m be given by (20). Given the numerator $m_{1}$ there is a best $=$ largest denominator $d\left(T, m_{1}\right)$ for which $m_{1}(B) / d\left(T, m_{1}\right)$ is a perturbation function of the same type $-\Phi, \Phi_{+}$, or $\Phi_{\alpha}-$ as $m$.

Given the denominator $m_{2}$, there is a best $=$ smallest numerator $n\left(B, m_{2}\right)$ for which $n\left(B, m_{2}\right) / m_{2}(T)$ is a perturbation function of the same type as $m$.

Proof. Suppose that $m$ is a $\Phi_{+}$p.f. Set

$$
d(T)=\inf \left\{m_{1}(B): T+B \text { is not } \Phi_{+}\right\} .
$$

We claim that $m_{1}(B) / d(T)$ is the minimal $\Phi_{+}$p.f. with numerator $m_{1}(B)$. First, if $m_{1}(B) / d(T)<1$, then by the definition of $d$ in (21), $T+B$ is $\Phi_{+}$. Second, suppose that $m_{1}(B) / d^{\prime}(T)$ is a $\Phi_{+}$p.f. Let a $\Phi_{+}$operator $T$ be given. For each $\varepsilon>0$ there is a $B$ with $d(T)+\varepsilon>m_{1}(B)$ and $T+B$ not $\Phi_{+}$; since $T+B$ is not $\Phi_{+}, m_{1}(B) / d^{\prime}(T) \geqslant 1$ and thus $d^{\prime}(T)<d(T)+\varepsilon$. Hence for each $\Phi_{+} T$, $d(T) \geqslant d^{\prime}(T)$. Note, in particular, that $d(T) \geqslant m_{2}(T)>0$.

To define $d(T)$ for a Fredholm p.f., replace $\Phi_{+}$.by $\Phi$ in (21). To define $d(T)$ for a $\Phi_{\alpha}$ p.f., set

$$
d(T)=\inf \left\{m_{1}(B): \text { either } T+B \text { is not } \Phi_{+} \text {or } \alpha(T+B)>\alpha(T)\right\} .
$$

In either case, proceed as above to see that the $d$ so defined is maximal.

Suppose, again, that $m$ is a $\Phi_{+}$p.f. To show that the best numerator, given $m_{2}$, exists, let $\mathscr{N}$ be the set of all those numerators $n^{\prime}$ for which $n^{\prime}(B) / m_{2}(T)$ is a $\Phi_{+}$p.f. and set

$$
n(B)=\inf \left\{n^{\prime}(B): n^{\prime} \text { is in } \mathscr{N}\right\} .
$$

If $n(B) / m_{2}(T)<1$, then there is an $n^{\prime}$ in $\mathscr{N}$ with $n^{\prime}(B) / m_{2}(T)<1$, hence $T+B$ is $\Phi_{+}$. That $n(B)$ is the smallest member of $\mathscr{N}$ and that $n(\lambda B)=|\lambda| n(B)$, follow from (22).

Note that one cnuld alternatively define $d(T)$ analogous to (22) or could define $n(B)$ analogous to (21).

Similar definitions and proofs establish the results for $\Phi$ and $\Phi_{\alpha}$ perturbation functions.

The notation $n\left(B, m_{2}\right)$ and $d\left(T, m_{1}\right)$ ambiguously denotes the numerator and denominator of either a $\Phi_{+}$, or $\Phi$, or $\Phi_{\alpha}$ perturbation function; context indicates which is meant.

Recall that Kato's strictly singular operators [9] contain the compact operators [6, pp. 84-5].

18. Lemma. Suppose that $m_{1}(B) / m_{2}(T)$ is either a $\Phi_{+}$or a $\Phi$ perturbation function. Let $n\left(B, m_{2}\right)=n(B)$ be the best numerator given the denominator $m_{2}$, and let $d\left(T, m_{1}\right)=d(T)$ be the best denominator given $m_{1}$, as discussed in 
Theorem 17. Then:

(i) $n(\lambda B)=|\lambda| n(B)$.

(ii) $d(\lambda T)=|\lambda| d(T)$.

(iii) $n(B+S)=n(B)$ for $S$ strictly singular.

(iv) $d(T+S)=d(T)$ for $S$ strictly singular.

Proof. Suppose that $m_{1} / m_{2}$ is a $\Phi_{+}$p.f. Property (i) is immediate. To see that (ii) holds,

$$
\begin{aligned}
d(\lambda T) & =\inf \left\{m(B): \lambda T+B \text { is not } \Phi_{+}\right\} \\
& =\inf \left\{m(\lambda B / \lambda): T+B / \lambda \text { is not } \Phi_{+}\right\}=|\lambda| d(T) .
\end{aligned}
$$

Define $n^{\prime}(B)=\inf \{n(B+S): S$ is strictly singular $\}$. If $n^{\prime}(B) / m_{2}(T)<1$, then there is a strictly singular $S$ with $T+B+S \Phi_{+}$and so $T+B$ is $\Phi_{+}[6$, p. 117]. Since $n^{\prime}(\lambda B)=|\lambda| n^{\prime}(B), n^{\prime}(B) / m_{2}(T)$ is a $\Phi_{+}$p.f., and therefore, by the minimality of $n, n^{\prime}=n$.

Define $d^{\prime}(T)=\sup \{d(T+S): S$ is strictly singular $\}$. As above, $m_{1}(B) / d^{\prime}(T)$ is a $\Phi_{+}$p.f., and by the maximality of $d, d^{\prime}=d$.

The proof for a Fredholm p.f. is similar.

Note that property (ii) also holds for a $\Phi_{\alpha}$ perturbation function.

19. DeFinition.

(i) $\mathrm{A} \Phi_{+}$perturbation function $m(B, T)$ is T-exact if given any $\Phi_{+}$ operator $T$ and $\varepsilon>0$ there is a $B$ with $m(B, T)<1+\varepsilon$, yet $T+B$ is not $\Phi_{+}$.

(ii) A Fredholm perturbation function $m(B, T)$ is $T$-exact if given any Fredholm operator $T$ and $\varepsilon>0$ there is a $B$ with $m(B, T)<1+\varepsilon$, yet $T+B$ is not Fredholm.

(iii) A $\Phi_{\alpha}$ perturbation function $m(B, T)$ is $T$-exact if given any $\Phi_{+}$ operator $T$ and $\varepsilon>0$ there is a $B$ with $m(B, T)<1+\varepsilon$, and yet either $T+B$ is not $\Phi_{+}$or $\alpha(T+B)>\alpha(T)$.

20. LEMMA. If $m_{1}(B) / m_{2}(T)$ is a T-exact perturbation function, then $m_{2}(T)$ is the best denominator, $d\left(T, m_{1}\right)$, given $m_{1}$.

Proof. See the proof of Theorem 17.

21. Corollary. Given the numerator $\|B\|$ :

(i) Kato's $\gamma(T)$ is the best denominator for a $\Phi_{\alpha}$ perturbation function.

(ii) The function $\mu(T)$ of Definition 2 is the best denominator for $a \Phi_{+}$ perturbation function.

(iii) $\mu(T)$ is also the best denominator for a $\Phi$ perturbation function.

Proof. Statements (i) and (ii) follow from Theorem 1, Theorem 4, and Lemma 20.

By Lemma $6,\|B\| / \mu(T)$ is also a $\Phi$ p.f., and since $\|B\| / \mu(T)$ is $T$-exact as a $\Phi_{+}$p.f., it is $T$-exact as a $\Phi$ p.f.
A $\Phi_{+}$perturbation function $m$ which has $m(T, T)=1$ for each $\Phi_{+}$ operator $T$ is $T$-exact, since $B=-T$ then satisfies $m(B, T)=1$ and $\alpha(T+B)=\infty$. Suppose that, in addition, the map $K \rightarrow m(K, T)$ is upper semicontinuous with respect to the norm topology on compact operators $K$. (Compare this with condition (ii) of Lemma 22 below.) It is easy to construct a compact $K$ with nonclosed range and infinite nullity $\alpha(K)$. Given $\varepsilon>0$, for small positive $\delta, B=-T+\delta K$ will have $m(B, T)<1+\varepsilon$ and yet have both $\alpha(T+B)=\infty$ and nonclosed range. This is apparently stronger than $m$ being $T$-exact. It holds for $\varrho(B, T)$, since the two conditions mentioned are easily shown to be satisfied by $\varrho$.

Compare (24) below with Definition 2.

22. LeMMA. Suppose that $m_{1}(B) / m_{2}(T)$ is a $\Phi_{+}$perturbation function.

(23) (i) $d\left(T, m_{1}\right)=\inf \left\{m_{1}(A+K): \alpha(T-A)=\infty\right.$ and $K$ compact $\}$.

(ii) If the map $K \rightarrow m_{1}(B+K)$ is upper semicontinuous with respect to the norm topology on compact operators $K$, then

$$
d\left(T, m_{1}\right)=\inf \left\{m_{1}(A): \alpha(T-A)=\infty\right\} .
$$

(iii) The best numerator, given the denominator of (23), is

$$
n\left(B, d\left(\cdot, m_{1}\right)\right)=\sup \left\{d\left(A, m_{1}\right): \alpha(B-A)=\infty\right\} .
$$

Proof. Let $d(T)$ be the R.H.S. of (23). If $T+B$ is not $\Phi_{+}$, by Lemma 3 there is a compact $K$ with $\alpha(T+B-K)=\infty$. Then $m_{1}(B)=m_{1}(B-K+K)$ $\geqslant d(T)$, and it follows that $m_{1}(B) / d(T)$ is a $\Phi_{+}$p.f. Given $\varepsilon>0$ there is an $A$ and a compact $K$ with $\alpha(T-A)=\infty$ and $m_{1}(A+K)<d(T)+\varepsilon$. Since $T-A$ is not $\Phi_{+}$, setting $B=A+K, T-B$ is also not $\Phi_{+}$and yet $m_{1}(B)<d(T)+\varepsilon$. Hence the p.f. $m_{1}(B) / d(T)$ is T-exact, and therefore $d(T)=d\left(T, m_{1}\right)$ by Lemma 20. This completes the proof of part (i).

Assume the condition of part (ii) holds. Given $\varepsilon>0$ there is an $A$ and compact $K$ with $\alpha(T-A)=\infty$ and $m_{1}(A+K)<d(T)+\varepsilon$. Since $T-A$ is not $\Phi_{+}, T-(A+K)$ is not $\Phi_{+}$, and so, given $\delta>0$, there is a compact $K^{\prime}$ with $\left\|K^{\prime}\right\|<\delta$ and $\alpha\left(T-\left(A+K+K^{\prime}\right)\right)=\infty$. Set $A^{\prime}=A+K+K^{\prime}$. Then $\alpha\left(T-A^{\prime}\right)$ $=\infty$ and $m_{1}\left(A^{\prime}\right)<m_{1}(A+K)+\varepsilon$ for small $\delta$. Hence $\inf \left\{m_{1}(B): \alpha(T-B)=\infty\right\}$ $<d(T)+2 \varepsilon$, from which (ii) follows.

It is useful to note that

$$
d(T)>0 \text { iff } T \text { is } \Phi_{+} .
$$

To see this, note that if $T$ is $\Phi_{+}$, then $d(T) \geqslant m_{2}(T)>0$, while if $T$ is not $\Phi_{+}$, then there is a compact $K^{\prime}$ with $\alpha\left(T-K^{\prime}\right)=\infty$ and from (23), $d(T)$ $\leqslant \inf \left\{m_{1}\left(K^{\prime}+K\right): K\right.$ compact $\}=0$.

Let $n(B)$ be the R.H.S. of $(25)$. Note that $n(B) \leqslant m_{1}(B)$. If $T+B$ is not $\Phi_{+}$, there is a compact $K$ with $\alpha(T+B+K)=\infty$. And then $n(B) \geqslant d(T+K)$ 
$=d(T)$, the last equality following from Lemma 18 , from which we see that $n(B) / d(T)$ is a $\Phi_{+}$p.f. Suppose that $n^{\prime}(B) / d(T)$ is a $\Phi_{+}$p.f., and assume that for some $B, n^{\prime}(B)<n(B)$. Using (25), given $0<\varepsilon<1$ there is a $T$ with $\alpha(B-T)=\infty$ and $d(T)>n(B)(1-\varepsilon)$. By $(26), T$ is $\Phi_{+}$. But then $n^{\prime}(B) / d(T)$ cannot be a $\Phi_{+}$p.f. after all, since for small enough $\varepsilon$ and $T$ and $B$ chosen as above, $n^{\prime}(B) / d(T)<1$ yet $T+B$ is not $\Phi_{+}$. Hence, $n(B)=n\left(B, d\left(\cdot, m_{1}\right)\right)$..

Definition. (i) A $\Phi_{+}$perturbation function $m(B, T)$ is $B$-exact if for each $B$ with the property that $m(B, T)$ is not identically zero for all $\Phi_{+} T$, given $\varepsilon>0$ there is a $T$ in $\Phi_{+}$with $m(B, T)<1+\varepsilon$ but $T+B$ not $\Phi_{+}$.

(ii) A Fredholm perturbation function $m(B, T)$ is $B$-exact if for each $B$ with the property that $m(B, T)$ is not identically zero for all Fredholm $T$, given $\varepsilon>0$ there is a Fredholm $T$ with $m(B, T)<1+\varepsilon$ but $T+B$ not Fredholm.

(iii) A $\Phi_{\alpha}$ perturbation function $m(B, T)$ is $B$-exact if for each $B$, given $\varepsilon>0$ there is a $T$ in $\Phi_{+}$with $m(B, T)<1+\varepsilon$ but either $T+B$ is not $\Phi_{+}$or $\alpha(T)>\alpha(T+B)$.

If $m(B, T)$ is a perturbation function and $B$ has the property that $m(B, T)=0$ for all $T$ in $\Phi_{+}$, then $T+B$ is $\Phi_{+}$for all $\Phi_{+} T$, i.e. such a $B$ is an admissible $\Phi_{+}$perturbation. An interesting result due to Weis $[18$, p. 429] is that in the case of operators mapping $a$ Banach space $X$ into itself, if $X$ is weakly compactly generated, then the set of $\Phi_{+}$admissible perturbations coincides with the strictly singular operators. (Not all Banach spaces are weakly compactly generated but all separable spaces and all reflexive spaces are [3]). Lemma 18 shows that for a given $\Phi_{+}$perturbation function $m_{1}(B) / m_{2}(T), n\left(S, m_{2}\right)=0$ for $S$ strictly singular; conversely, if $X$ is weakly compactly generated, then $n\left(S, m_{2}\right)=0$ implies, by the above, that $S$ is strictly singular.

24. LEMMA. If $m_{1}(B) / m_{2}(T)$ is a B-exact perturbation function, then $m_{1}(B)$ is the best numerator $n\left(B, m_{2}\right)$ given the denominator $m_{2}$.

Proof. See the last paragraph of the proof of Lemma 22.

Begin with a perturbation function $m_{1}(B) / m_{2}(T)$. Then, given $m_{1}$, the best denominator given $m_{1}, d\left(T, m_{1}\right)$, can be found. Next, one can find the best numerator $n(B, d)$ given that denominator $d(T)=d\left(T, m_{1}\right)$. These numerators and denominators are described in Lemma 22 for $\Phi_{+}$perturbation functions, for example. One could conceivably continue this process indefinitely: next getting a better denominator, given the numerator $n(B, d)$, then a better numerator given this new denominator, and so on. It will be shown in Theorem 27 that this process actually stops with $d(T)$ and $n(B, d)$.

Let a factored perturbation function $m_{1}(B) / m_{2}(T)$ be given. Since, for a positive constant $a, a m_{1}(B) /\left(a m_{2}(T)\right)$ is the same function, to fix this constant $a$ it will be supposed that there is a given $\Phi_{+}$or $\Phi$ operator $T_{0}$ with $m_{2}\left(T_{0}\right)=1$, and this will also be required of the denominator of any improvement made to the perturbation function. For operators mapping $X$ into itself a natural choice is to take $T_{0}$ to be the identity. In fact, it will be useful to require a slightly more restrictive normalization, given in Definition 25 below.

25. Definrmion. A factored perturbation function $m_{1}(B) / m_{2}(T)$, to which the condition $m_{2}\left(T_{0}\right)=1$ is applied, is normalized at $T_{0}$ if, in addition, $m_{1}\left(T_{0}\right)=1$.

26. LeMMA. Let $m_{1}(B) / m_{2}(T)$ be a perturbation function normalized at $T_{0}$. Suppose that $m_{1}^{\prime}(B) / m_{2}^{\prime}(T)$ is a smaller perturbation function, i.e. $m_{1}^{\prime}(B) / m_{2}^{\prime}(T)$ $\leqslant m_{1}(B) / m_{2}(T)$ for all $B$ and all suitable T. Here, without loss of generality, we take $m_{2}^{\prime}\left(T_{0}\right)=1$.

Then $m_{1}^{\prime}(B) \leqslant m_{1}(B)$ for all $B$, and $m_{2}^{\prime}(T) \geqslant m_{2}(T)$ for all appropriate $T$.

Proof. Choosing $T=T_{0}$ shows that we have $m_{1}^{\prime}(B) \leqslant m_{1}(B)$. Since $m_{1}^{\prime}\left(T_{0}\right) / m_{2}^{\prime}\left(T_{0}\right) \leqslant m_{1}\left(T_{0}\right) / m_{2}\left(T_{0}\right)=1$, and since $m_{1}^{\prime}\left(T_{0}\right) / m_{2}^{\prime}\left(T_{0}\right) \geqslant 1$, because $T_{0}+\left(-T_{0}\right)$ is not $\Phi_{+}$, it must be that $m_{1}^{\prime}\left(T_{0}\right)=1$. Taking $B=T_{0}$ shows that $m_{2}^{\prime}(T) \geqslant m_{2}(T)$.

27. THEOREM. Suppose that $m_{1}(B) / m_{2}(T)$ is a perturbation function normalized at $T_{0}$. Then there is no perturbation function smaller than $n\left(B, d\left(\cdot, m_{1}\right)\right) / d\left(T, m_{1}\right)$.

Proof. Suppose that $m_{1}^{\prime}(B) / m_{2}^{\prime}(T)$ is a p.f. less than or equal to $m_{1}(B) / m_{2}(T)$ for all $B$ and all suitable $T$. Without loss of generality, $m_{2}^{\prime}\left(T_{0}\right)=1$. By Lemma $26, m_{2}^{\prime}(T) \geqslant m_{2}(T)$. Consequently, $m_{1}^{\prime}(B) / m_{2}^{\prime}(T) \leqslant m_{1}^{\prime}(B) / m_{2}(T)$, and this implies that the R.H.S. is a p.f., from which it follows that $m_{1}^{\prime}(B) \geqslant n\left(B, m_{2}\right)$. Since $m_{1}^{\prime}(B) \leqslant m_{1}(B), m_{1}^{\prime}(B) / m_{2}^{\prime}(T) \leqslant m_{1}(B) / m_{2}^{\prime}(T)$. This implies that the R.H.S. is a p.f. and, as above, this implies that $m_{2}^{\prime}(T)$ $\leqslant d\left(T, m_{1}\right)$. Hence

$$
\begin{aligned}
& n\left(B, m_{2}\right) \leqslant m_{1}^{\prime}(B) \leqslant m_{1}(B), \\
& m_{2}(T) \leqslant m_{2}^{\prime}(T) \leqslant d\left(T, m_{1}\right) .
\end{aligned}
$$

Suppose that $m_{1}^{\prime}(B) / m_{2}^{\prime}(T) \leqslant m_{1}(B) / d\left(T, m_{1}\right)$. By $(28), m_{2}^{\prime}(T)=d\left(T, m_{1}\right)$. By (27), $n\left(B, d\left(\cdot, m_{2}\right)\right) / d\left(T, m_{2}\right) \leqslant m_{1}^{\prime}(B) / m_{2}^{\prime}(T)$ and we have shown somewhat more than the theorem states.

For operators on a Banach space $X,\|B\| / \mu(T)$ is normalized at the identity. From Corollary $21, \mu(T)=d(T,\|\cdot\|)$. Thus the proof of Theorem 27 shows that the smallest $\Phi_{+}$perturbation function less than or equal to $\|B\| / \mu(T)$ has denominator $\mu(T)$ and numerator $n(B)$ given, as in Lemma 22, by $n(B)=\{\mu(A): \alpha(B-A)=\infty\}$.

28. THEOREM. For operators mapping a Hilbert space into itself:

(i) There is no factored $\Phi_{\alpha}$ perturbation function less than $\|B\| / \gamma(T)$.

(ii) There is no factored $\Phi_{+}$or $\Phi$ perturbation function less than $\Delta(B) / \Gamma(T)$. 
Proof. Let a nonzero $B$ be given. For $0<\varepsilon<\|B\|$, there is a $v$ of norm one with $\|B v\|>\|B\|-\varepsilon$. Let $U$ be an isometry of the orthogonal complement of $\operatorname{sp}(v)$ onto the orthogonal complement of $\operatorname{sp}(v, B v)$. Define $T$ by $T=-B$ on $\operatorname{sp}(v)$ and $T=\lambda U$ on $\operatorname{sp}(v)^{\perp}$, where $\lambda=\|B\|-\varepsilon$. Given $x$, let $a v$ be the projection of $x$ onto $\operatorname{sp}(v)$. Then $\|T x\|^{2}=|a|^{2}\|B v\|^{2}+\lambda^{2}\|x-a v\|^{2} \geqslant \lambda^{2}\|x\|^{2}$. For this Fredholm $T,\|B\| \leqslant \gamma(T)+\varepsilon$, but $\alpha(T+B) \geqslant 1>\alpha(T)$. Hence $\|B\| / \gamma(T)$ is $B$-exact as a $\Phi_{\alpha}$ p.f., and, by Lemma $24,\|B\|$ is the best $\Phi_{\alpha}$ numerator given $\gamma(T)$. By Corollary 21, $\gamma(T)$ is the best $\Phi_{\alpha}$ denominator given $\|B\|$, and (i) follows from the proof of Theorem 27.

From Definition 2 and (6) it follows that, on a Hilbert space, $\mu(T)=\Gamma(T)$, and then Corollary 21 shows that $\Gamma(T)=d(T,\|\cdot\|)$, either as a $\Phi_{+}$or as a $\Phi$ p.f.

Suppose that $B$ is not strictly singular (which is the same as compact on a Hilbert space). Given $0<\varepsilon<\Delta(B)$, there is a subspace $M$ on which $\inf \left\{\left\|\left.B\right|_{N}\right\|: N \subset M\right\}>\Delta(B)-\varepsilon$. Then $\Gamma\left(\left.B\right|_{M}\right)>0$ and so $\left.B\right|_{M}$ is $\Phi_{+}$, which implies that $v\left(\left.B\right|_{M}\right)>0$. By Lemma 13 , there is an $M^{\prime} \subset M$ with $\left\|\left.B\right|_{M^{\prime}}\right\|<j\left(\left.B\right|_{M^{\prime}}\right)+\varepsilon$.

Let $\operatorname{dim}$ denote the Hilbert space dimension. If $\operatorname{dim} M^{\prime}<\operatorname{dim} H, H$ the Hilbert space, then $\operatorname{dim}\left(M^{\prime}\right)^{\perp}=\operatorname{dim}\left(B M^{\prime}\right)^{\perp}$. If $\operatorname{dim} M^{\prime}=\operatorname{dim} H$, there is an $M^{\prime \prime} \subset M^{\prime}$ with $\operatorname{dim} M^{\prime \prime}=\operatorname{dim}\left(M^{\prime \prime}\right)^{\perp}=\operatorname{dim} H$, and $\operatorname{dim}\left(M^{\prime \prime}\right)^{\perp}=\operatorname{dim}\left(B M^{\prime \prime}\right)^{\perp}$. In either case there is an $M^{\prime \prime} \subset M^{\prime}$ and an isometry $U$ of $\left(M^{\prime \prime}\right)^{\perp}$ onto $\left(B M^{\prime \prime}\right)^{\perp}$. Define $T$ by $T=-B$ on $M^{\prime \prime}$ and $T=\lambda U$ on $\left(M^{\prime \prime}\right)^{\perp}$, where $\lambda=j\left(\left.B\right|_{M^{\prime}}\right)$. Then, writing any $x=x^{\prime \prime}+y, x^{\prime \prime}$ in $M^{\prime \prime}$ and $y$ in $\left(M^{\prime \prime}\right)^{\perp},\|T x\|^{2}=\left\|B x^{\prime \prime}\right\|^{2}+\lambda^{2}\|y\|^{2} \geqslant$ $\lambda^{2}\|x\|^{2}$. Hence on any subspace $N,\left\|\left.T\right|_{N}\right\| \geqslant \lambda \geqslant\left\|\left.B\right|_{\mathcal{M}^{\prime \prime}}\right\|-\varepsilon>\Delta(B)-2 \varepsilon$. Thus $\Delta(B)<\Gamma(T)+2 \varepsilon$, yet for the $\Phi$ operator $T, \alpha(T+B)=\infty$. Then $\Delta(B) / \Gamma(T)$ is $B$-exact, as either a $\Phi$ or a $\Phi_{\alpha}$ p.f., and by Lemma $24, \Delta(B)=n(B, \Gamma)$, and (ii) follows from Theorem 27.

Let $A$ map $X$ into itself. Note that for the function appearing in Theorem 11

$$
r_{\sigma}(\pi(A))=\lim \left\|\pi\left(A^{n}\right)\right\|^{1 / n}=\sup \{|\lambda|: A-\lambda \text { is not } \Phi\} .
$$

A related function is

$$
r_{+}(A)=\inf \left\{|\lambda|: A-\lambda \text { is not } \Phi_{+}\right\} .
$$

29. Lemma. Consider operators from $X$ into itself and let $m_{1}$ and $m_{2}$ be functions from these operators to $\boldsymbol{R}^{+}$satisfying:

(a) $m_{j}(I)=1, j=1,2$.

(b) $m_{j}(\lambda A)=|\lambda| m_{j}(A), \quad j=1,2$.

(c) Whenever $T$ is a $\Phi_{+}$operator, $m_{1}(B)<m_{2}(T)$, and $B$ commuies'with $T$, then $T+B$ is a $\Phi_{+}$operator.

Then for all $A$,

$$
\begin{gathered}
r_{\sigma}(\pi(A)) \leqslant m_{1}(A), \\
r_{+}(A) \geqslant m_{2}(A) .
\end{gathered}
$$

Proof. For $m_{1}(A)<|\lambda|=m_{2}(\lambda I), A-\lambda I$ is $\Phi_{+}$. As in the proof of Lemma $6, x(a A-\lambda I)=x(\lambda I)$ for $a$ in $[0,1]$, thus $A-\lambda I$ is Fredholm, from which (31) follows. On the other hand, if $m_{1}(\lambda I)=|\lambda|<m_{2}(A)$ then $A-\lambda I$ is $\Phi_{+}$, from which (32) follows.

Replacing $\Phi_{+}$by $\Phi$ in condition (c) above gives a related lemma with (32) replaced by $r_{\sigma}(\pi(A)) \geqslant m_{2}(A)$.

30. THEOREM. Consider operators mapping $X$ into itself. Suppose that

$$
r_{\sigma}(\pi(B))<r_{+}(T)
$$

and that $T$ and $B$ almost commute in the sense that $T B-B T$ is compact. Then $T+B$ is $\Phi_{+}$. In addition, $\chi(T+B)=\chi(T)$, so that if $T$ is $\Phi$ then so is $T+B$.

Proof. Implicit in (33) is $r_{+}(T)>0$ which implies that $T$ is $\Phi_{+}$. A result proved in [22] (see also [17] and [4]) is $r_{+}(T)=\lim \left(\Gamma\left(T^{n}\right)\right)^{1 / n}$. From (33), there is an $n$ with $\left\|\pi\left(B^{n}\right)\right\|<\Gamma\left(T^{n}\right)$. Thus there is a compact operator $K$ with $\left\|B^{n}-K\right\|<\Gamma\left(T^{n}\right)$. Hence $T^{n}+B^{n}-K$ is $\Phi_{+}$and therefore so is $T^{n}+B^{n}$. Using the hypothesis that $T$ and $B$ almost commute, for $A=T^{n-1}-B T^{n-2}+\ldots$, $T^{n}+B^{n}=(T+B) A+K_{1}=A(T+B)+K_{2}$, where $K_{1}$ and $K_{2}$ are compact. Thus $A(T+B)$ and $(T+B) A$ are both $\Phi_{+}$and therefore so is $T+B$.

The argument above shows that $T+\lambda B$ is $\Phi_{+}$for $\lambda$ in $[0,1]$ : as in the proof of Lemma $6, \varkappa(T+B)=\varkappa(T)$. Consequently, $T+B$ is Fredholm if $T$ is Fredholm.

With the appropriate definitions, which are left to the reader, Lemma 29 and Theorem 30 show that $r_{\sigma}(\pi(B)) / r_{+}(T)$ is the smallest factored (almost) commuting $\Phi_{+}$perturbation function normalized at the identity.

The results here can be extended to the perturbation of $\Phi_{-}$operators and, in the obvious notation, $\Phi_{-}$and $\Phi_{\beta}$ perturbation functions.

\section{References}

[1] S. Banach, Théorie des Opérations Linéaires, Warszawa 1932; reprinted by Chelsea New York 1955.

[2] C. Bessaga and A. Pełczyński, Banach spaces non-isomorphic to their Cartesian squares. I, Bull. Acad. Polon. Sci. 8 (1960), 77-80.

[3] J. Dieste1, Geometry of Banach Spaces-Selected Topics, Lecture Notes in Math. 485, Springer, New York 1975.

[4] A. Fainshtein, On measures of noncompactness of linear operators and analogues of the minimum modulus for semi-Fredholm operators, Spektr. Teor. Operat. i Prilozhen. No. 6 (1985), 182-195 (in Russian). 
[5] I. Feldman, I. Gohberg and A. Markus, Normally solvable operators and ideals associated with them, Izv. Moldavsk. Filiala Akad. Nauk SSSR 1960, no. 10, 51-70; AMS Transl. 61 (1967), 63-84.

[6] S. Goldberg, Unbounded Linear Operators, McGraw-Hill, New York 1966.

[7] I. Gohberg and M. Krein, Fundamental theorems on deficiency numbers, root numbers, and indices of linear operators, Uspekhi Mat. Nauk 12(2) (1957), 43-118; AMS Transl. Series 2, Vol. 13.

[8] K. Jörgens, Linear Integral Operators, Pitman, London 1982.

[9] T. Kato, Perturbation theory for nullity, deficiency, and other quantities of linear operators, J. Analyse Math. 6 (1958), 261-322.

[10] - Perturbation Theory for Linear Operators, Springer, New York 1966.

[11] E. Makai, Jr. and J. Zemánek, The surjectivity radius, packing numbers and boundedness below of linear operators, Integral Equations Operator Theory 6 (1983), 372 384.

[12] A. Pełczyński, On strictly singular and strictly cosingular operators. I. Strictly singular and strictly cosingular operators in C(S)-spaces, Bull. Acad. Polon. Sci. 13 (1965), 31-36,

[13] A. Pietsch, Operator Ideals, North-Holland, Amsterdam 1980

[14] M. Schechter, Quantities related to strictly singular operators, Indiana Univ. Math. J. 21 (1972), 1061-1071.

[15] -, Riesz operators and Fredholm perturbations, Bull. Amer. Math. Soc. 74 (1968), 1139--1144.

$[16]$ -, Principles of Functional Analysis, Academic Press, New York 1971.

[17] H.-O. Tylli, On the asymptotic behaviour of some quantities related to semiFredholm operators, J. London Math. Soc. 31 (1985), 340-348.

[18] L. Weis, Perturbation classes of semi-Fredholm operators, Math. Z. 178 (1981), 429-442.

[19] R. Whitley, Strictly singular operators and their conjugates, Trans. Amer. Math. Soc. 13 (1964), 252-261.

[20] K. Y line n, Measures of noncompactness for elements of $C^{*}$-algebras, Ann. Acad. Sci. Fenn. Ser. A 6 (1981), 131-133.

[21] B. Yood, Properties of linear transformations preserved under the addition of a completely continuous transformation, Duke Math. J. 18 (1951), 599-612.

[22] J, Zemánek, Geometric characteristics of semi-Fredholm operators and their asymptotic behaviour, Studia Math. 80 (1984), 219-234.

[23] -, The semi-Fredholm radius of a linear operator, Bull. Polish Acad. Sci. 32 (1984), 67-76.

[24] - On the A-characteristic of $M$. Schechter, in Proc. 2nd Internat. Conf. on Operator Algebras, Ideals, and their Appl. in Theor. Physics, H. Baumgärtel et al. (eds.), Teubner, Leipzig 1984, 232-234.

[25] -, The stability radius of a semi-Fredholm operator, Integral Equations Operator Theory 8 (1985), 137-144.

\section{UNIVERSITY OF CALIFORNIA AT IRVINE}

Irvine, California 92717, USA.

\section{On uncountable unconditional bases in Banach spaces}

by

LECH DREWNOWSKI (Poznań and East Lansing, Mich.)

Abstract. If a Banach space with an uncountable unconditional basis $\left(v_{j}\right)$ contains an isomorphic copy of $l_{1}(A)$ or $c_{0}(A)$ for some uncountable set $A$, then the basis $\left(v_{j}\right)$ has "large" subbases of $l_{1}$-type, or $c_{0}$-type, respectively (Theorems 1 and 2 ). This generalizes the results obtained by S. L. Troyanski in 1975 for Banach spaces with symmetric bases.

In Theorems 1 and 2 below, we extend to Banach spaces with uncountable unconditional bases the following result of Troyanski [6, Corollaries 1 and 2]:

Let $F$ be a Banach space with a symmetric basis $\left(v_{j}\right)_{j \in J}$. If $F$ has a subspace isomorphic to the Banach space $l_{1}(A)$ [resp., $c_{0}(A)$ ] for some uncountable set $A$, then the basis $\left(v_{j}\right)_{j \in J}$ is equivalent to the natural basis of the space $l_{1}(J)$ [resp., $\left.c_{0}(J)\right]$.

Our results show that if the basis $\left(v_{j}\right)_{j \in J}$ is merely unconditional, then it must contain large $l_{1}$ - [resp., $c_{0^{-}}$] subbases. Unlike in [6], where the above result was obtained via some renorming considerations, our arguments will be purely combinatorial. The $l_{1}$ part of Troyanski's result plays a crucial role in the author's recent paper [1]; the present work is, in a sense, a continuation of [1].

In general, our Banach space terminology and notation is that of [4] and [5].

Throughout, $F$ will be a (nonseparable) Banach space with an uncountable unconditional basis $\left(v_{j}\right)_{j \in J}$. Recall (cf. [5], [6]) that this means that for every $y$ in $F$ there is a unique family of scalars $\left(t_{j}\right)_{j \in J}$ such that $y=\sum_{j \in J} t_{j} v_{j}$ (unconditional convergence or summability). Let $\left(v_{j}^{*}\right)_{j \in J} \subset F^{*}$ be the dual family, biorthogonal to $\left(v_{j}\right)_{j \in J}$. Then, for $y$ in $F$, we define the support of $y$ as

$$
s(y) \doteq\left\{j \in J: v_{j}^{*}(y) \neq 0\right\}
$$

clearly, $|s(y)| \leqslant \aleph_{0} .(|A|$ denotes the cardinal number of the set $A$.) The natural unit vector bases in the spaces $l_{1}(A)$ and $c_{0}(A)$ will be denoted by $\left(e_{\alpha}^{1}\right)_{\alpha \in A}$ and

\footnotetext{
1980 Mathematics Subject Classification: Primary 46B15, 46B25. basis.

Key words and phrases: Banach space, uncountable unconditional basis, $l_{1}$-subbasis, $c_{0}$-sub.

2 - Studia Math, 90.3
} 